\title{
Composição florística e estrutura da comunidade de trepadeiras da Floresta Atlântica no Sul de Santa Catarina, Brasil
}

\author{
Lislaine Cardoso de Oliveira-Gomes', Jaqueline Durigon ${ }^{2}$, \\ Peterson Teodoro Padilha' \& Vanilde Citadini-Zanette'
}

\author{
1 Universidade do Extremo Sul Catarinense, Programa de Pós-Graduação em Ciências Ambientais, Av. Universitária, 1 105, CEP 88806-000, \\ Criciúma, Santa Catarina. lisoliveira@hotmail.com, peterpadilha@hotmail.com, vcz@unesc.net \\ 2 Universidade Federal do Rio Grande, Conselho de Ensino, Pesquisa e Extensão, \\ Av. Marechal Floriano, 2236, CEP 96170-000, São Lourenço do Sul, Rio Grande do Sul. jaquelinedurigon@gmail.com
}

Recebido em 13.VI.2016

Aceito em 11.1.2018

DOI 10.21826/2446-8231201873101

\begin{abstract}
RESUMO - As trepadeiras são importantes na composição e estrutura das florestas tropicais e subtropicais. No presente estudo foi realizado levantamento florístico e fitossociológico das comunidades de trepadeiras no Parque Estadual da Serra Furada, Sul de Santa Catarina, Brasil. A flora local foi amostrada por meio de caminhamento exploratório, em áreas alteradas, enquanto que os dados estruturais foram registrados em áreas do interior da floresta. Foram amostradas 70 espécies, sendo 44 lenhosas e 26 herbáceas. As famílias de maior riqueza específica foram Asteraceae (14), Bignoniaceae (12) e Apocynaceae (oito). A estratégia de dispersão mais frequente foi anemocoria. Mickelia scandens (Raddi) R.C. Moran foi a espécie mais abundante e com maior Índice de Valor de Importância. O mecanismo de escalada com maior ocorrência foi o volúvel, enquanto em abundância o radicante. O estudo traz contribuições ao conhecimento da flora de trepadeiras do Sul do Brasil, visto que inclui as trepadeiras herbáceas, que raramente estão em estudos quantitativos deste grupo de plantas.
\end{abstract}

Palavras-chave: herbácea, lenhosa, mecanismo de escalada

ABSTRACT - Floristic composition and community structure of climbers from the Atlantic Rain Forest in southern Santa Catarina, Brazil. Climbers are important for the composition and structure of tropical and subtropical forests. This study aimed to analyze the floristic and community structure survey of climbers in the Parque Estadual da Serra Furada, in southern Santa Catarina, Brazil. The floristic survey was carried out in disturbed areas by the walking method, and the structural survey was conducted in inland areas of the forest. Seventy species of climbers were recorded, 44 were woody and 26 were herbaceous. The most representative families were Asteraceae (14), Bignoniaceae (12) and Apocynaceae (eight). Anemochory was the most common dispersion strategy. Mickelia scandens (Raddi) R.C. Moran was the most abundant species and had the highest Importance Value Index. Voluble was the climbing mechanism most found, while radicant was most abundant. This study contributes to the knowledge of vine flora from southern Brazil, including herbaceous climbers, rarely included in quantitative studies of this plant group.

Keywords: climbing mechanism, herbaceous, woody

\section{INTRODUÇÃO}

A Mata Atlântica é um bioma que agrega grande diversidade e endemismo no Brasil. No entanto, com a ocorrência de grande degradação e retirada da cobertura vegetal, restam em torno de $7 \%$ da sua composição original (Lagos \& Muller 2007). No estado de Santa Catarina, que está completamente inserido na Mata Atlântica, este percentual chega a menos de $5 \%$ da vegetação (Campanili \& Schäffer 2010). A Floresta Ombrófila Densa, um dos tipos de vegetação que compõe este bioma, cobre cerca de $40 \%$ do Estado, e está atualmente representada por remanescentes isolados de diferentes tamanhos e estágios sucessionais, resultado em grande parte da ação antrópica sobre a floresta (Vibrans et al. 2013).

As trepadeiras são elementos importantes da composição e estrutura da flora tropical e subtropical (Schnitzer \& Bongers 2002). Na Mata Atlântica e em outras florestas ombrófilas brasileiras encontram as condições ambientais propícias ao seu estabelecimento, compondo de forma expressiva a flora local (CitadiniZanette et al. 2014).

As trepadeiras são plantas mecanicamente dependentes que necessitam de um suporte (forófito) para seu estabelecimento e ascensão ao dossel, e que se mantêm enraizadas no solo durante toda a vida (Gentry 1991). As trepadeiras lenhosas (lianas) são as trepadeiras mais estudadas e incluídas em análises quantitativas. Elas possuem maior diâmetro e são mais comuns no interior da floresta, geralmente atingindo o dossel da mesma. Já as trepadeiras herbáceas (vinhas), apresentam caule de pequena espessura ou sublenhosos e ocorrem, geralmente, em áreas mais alteradas e bordas de florestas ou, ainda, no sub-bosque (Gentry 1991).

Devido a maior atenção dada às trepadeiras lenhosas, muito pouco se sabe sobre a florística e a ecologia das herbáceas. As trepadeiras herbáceas geralmente são excluídas de estudos quantitativos, sendo seu conhecimento escasso quanto aos diversos parâmetros ecológicos comunitários, como abundância nas florestas. Entre os 
fatores que explicam a ausência deste grupo de plantas nos levantamentos, está o critério de inclusão considerando um diâmetro mínimo (Villagra 2012). Além disso, há um maior interesse no conhecimento dos fatores que influenciam a distribuição e abundância das trepadeiras lenhosas, em função dos vários estudos que mostram sua relação de competição com as árvores, sua grande influência na dinâmica local e global dos ecossistemas (Putz 1984, Tabanez \& Viana 2000, Phillips et al. 2002, Sfair et al. 2015). Por isso, as lianas têm sido alvo de muitos estudos, sendo que, a compreensão da diversidade e ecologia deste grupo, frente às alterações dos ambientes, se faz cada vez mais importante (Phillips et al. 2002, Durigon 2014, Sfair et al. 2015).

No Brasil, o estudo das plantas trepadeiras é recente, principalmente com a inclusão das trepadeiras herbáceas. A maioria dos estudos têm sido realizados em Florestas Estacionais Semideciduais (Rezende \& Weiser 2014), necessitando ainda de maior conhecimento em outras tipologias vegetacionais, como as florestas subtropicais.

O presente estudo tem por objetivo contribuir para o conhecimento da composição florística e estrutura das comunidades de trepadeiras das florestas ombrófilas brasileiras. Propõe-se um estudo qualitativo e quantitativo das trepadeiras lenhosas e herbáceas em uma área de Floresta Ombrófila Densa, localizada na região subtropical do Brasil.

\section{MATERIAL E MÉTODOS}

\section{Caracterização da área de estudo}

O estudo foi realizado no Parque Estadual da Serra Furada (PAESF), no sul do estado de Santa Catarina, (49 $25^{\prime} 17^{\prime \prime}$ e $49^{\circ} 22^{\prime} 58^{\prime \prime}$ de longitude Oeste e $28^{\circ} 08^{\prime} 13$ ' e $28^{\circ} 11^{\prime} 36^{\prime \prime}$ de latitude Sul). Com área de 1.344 ha que integra os municípios de Orleans e Grão-Pará, o Parque é uma Unidade de Conservação de Proteção Integral e foi criado em 20 de junho de 1980, por meio do Decreto $n^{\circ}$ 11.233 (FATMA 2010).

O clima da região, segundo Köppen, é do tipo Cfa. A temperatura média anual é de $18,7^{\circ} \mathrm{C}$, conforme dados da Estação Meteorológica de Orleans (EPAGRI 2001). Nas áreas mais elevadas, próximas do Planalto Catarinense, o clima é classificado como $\mathrm{Cfb}$. $\mathrm{Na}$ região onde está localizado o PAESF, os totais anuais precipitados estão em torno de 1650 e $1750 \mathrm{~mm} /$ ano e 130 dias de chuva/ano, onde as maiores precipitações ocorrem entre os meses de janeiro e março (FATMA 2010).

A geomorfologia do PAESF compreende três unidades: Patamares da Serra Geral, onde se encontra grande parte do Parque em que está inserida a Floresta Ombrófila Densa; Serra Geral, que se encontra na área de maior altitude e Depressão da Zona Carbonífera Catarinense, com a parte da Floresta Ombrófila Densa com altitudes abaixo de 580 metros, nas áreas antropizadas, áreas de estágio inicial de sucessão vegetal (FATMA 2010).
O Parque faz parte do bioma Mata Atlântica e é composto pela Floresta Ombrófila Densa (Montana e Altomontana) (IBGE 2012), sendo este estudo desenvolvido na sua área Montana.

\section{Levantamento fitossociológico}

Para o levantamento fitossociológico utilizou-se o método de parcelas (Mueller-Dombois \& Ellenberg 2002). Este foi conduzido com a demarcação de seis unidades amostrais de 20 x $100 \mathrm{~m}$. Dentro de cada unidade amostral foram sorteadas, aleatoriamente, 10 parcelas de $10 \times 10$ $\mathrm{m}$ para a amostragem, totalizando 60 parcelas, sendo a primeira unidade amostral estabelecida a cinco metros da borda da floresta. Este ambiente de interior de floresta possui características de estágio médio (unidades A e B) e avançado (C, D, E e F) de sucessão natural (Fig. 1).

Foram registradas todas as trepadeiras (herbáceas e lenhosas) que possuíam DAP igual ou superior a 0,2 $\mathrm{cm}$, enraizadas dentro da parcela, com altura mínima do caule principal de 1,30 m a partir do solo. As medições seguiram o protocolo padrão proposto por Gerwinget et al. (2006), onde as ramificações que enraízam e ascendem ao dossel, possuindo folhas, foram contadas como indivíduos independentes. Foram incluídas as samambaias e excluídos os bambus.

A lista florística da área de estudo incluiu as espécies registradas nas parcelas amostradas no interior da floresta, e também as encontradas fora das parcelas em áreas alteradas de floresta em estágio inicial e área antropizada na borda da floresta, sendo realizado caminhamento exploratório nestas áreas (Filgueiras et al. 1994). As espécies foram caracterizadas quanto aos mecanismos de escalada no forófito seguindo a classificação de Hegarty (1991). As estratégias de dispersão das trepadeiras foram registradas com base na literatura (Romaniuc-Neto et al. 2012, Santos et al. 2014).

A identificação botânica das espécies foi realizada por meio de chaves dicotômicas (Reitz 1968, 1974, 1980, Reis 2004, Villagra 2008), consulta ao Herbário Pe. Dr. Raulino Reitz (CRI) da Universidade do Extremo Sul Catarinense (UNESC) e Herbário do Instituto de Biociências (ICN) da Universidade Federal do Rio Grande do Sul, além de auxílio de especialistas. Os materiais testemunhos foram incorporados ao acervo do Herbário CRI.

O sistema de classificação adotado para famílias seguiu APG IV (2016) para angiospermas e Smith et al. (2006) para as samambaias. Os nomes das espécies foram revisados quanto à nomenclatura e abreviatura dos autores através da base de dados The International Plant Names Index (IPNI 2015).

\section{RESULTADOS E DISCUSSÃO}

Foram registradas 70 espécies de trepadeiras, distribuídas em 27 famílias e 47 gêneros, sendo 44 espécies lenhosas e 26 herbáceas (Quadro 1). Destas, 38 espécies foram amostradas no levantamento fitossociológico, que 


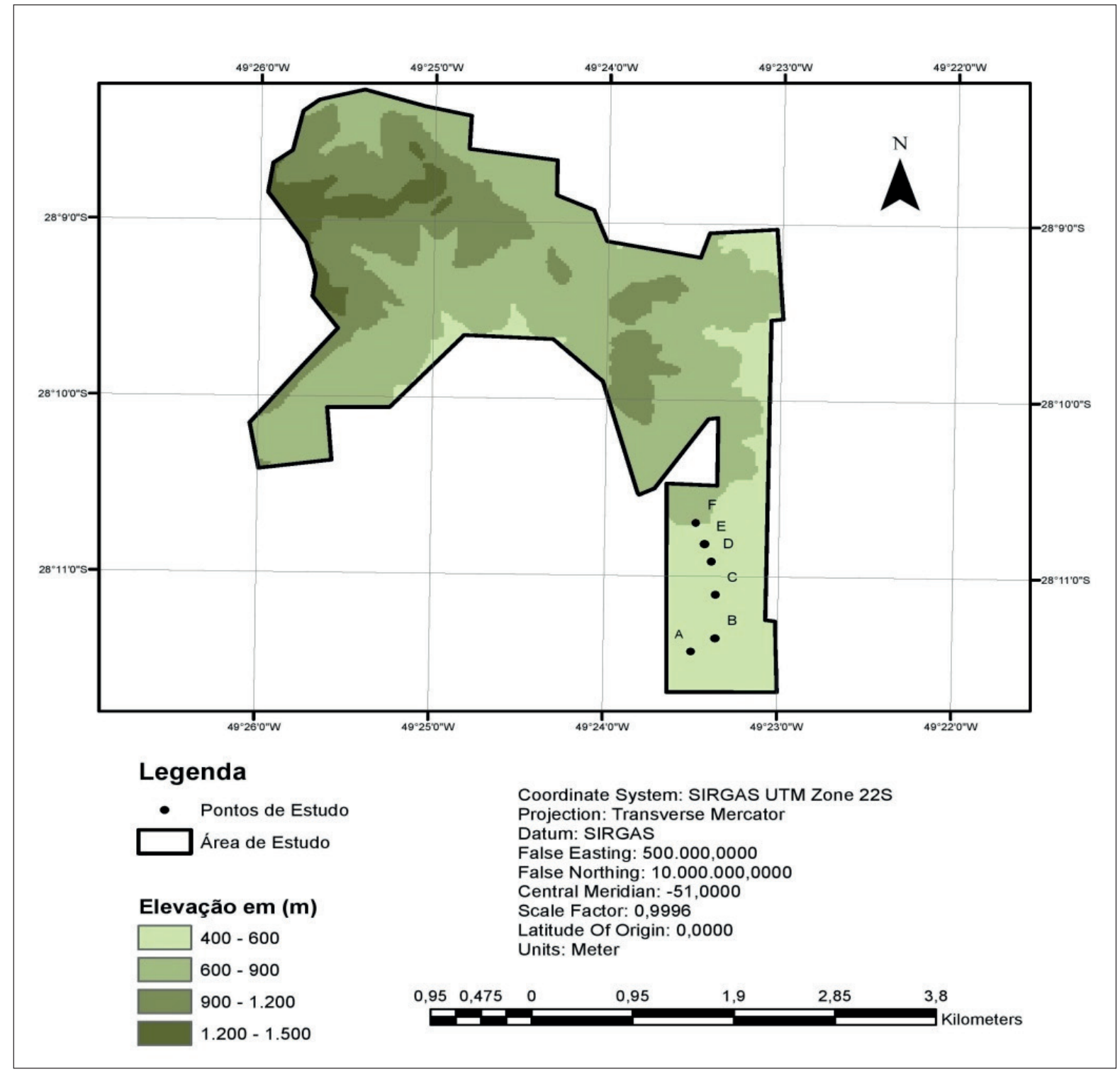

incluiu apenas as espécies do interior da floresta. A maior parte da riqueza de trepadeiras herbáceas, cerca de $62 \%$, foi encontrada no levantamento florístico por caminhamento, que explorou as áreas mais alteradas. A prevalência de trepadeiras lenhosas em ambientes florestais é comum (Udulutsch et at. 2004, Carneiro \& Vieira 2012, Villagra 2012), já em ambientes mais abertos ou alterados tem se evidenciado maior ocorrência de trepadeiras herbáceas (Gentry 1991, Durigon et al. 2009).

As famílias com maior riqueza foram Asteraceae com 14 espécies, Bignoniaceae com 12 e Apocynaceae com oito. O gênero mais presente foi Mikania Willd., com nove espécies. Estas famílias estão comumente entre as mais ricas nos levantamentos com trepadeiras no sul do Brasil (Durigon et al. 2009, Durigon \& Waechter 2011, Citadini-Zanette et al. 2014). Durigon (2010) atribui à dispersão anemocórica a ampla distribuição das espécies destas famílias em diferentes ambientes, sendo esta estratégia também a mais representativa neste estudo, estando representada por $70 \%$ do total de espécies amostradas (Quadro 1).

A riqueza de trepadeiras de Asteraceae é expressiva em ambientes de alta pluviosidade e de maior preservação (Villagra 2012), estando ainda entre as famílias mais ricas em espécies trepadeiras da região neotropical, ocupando o quarto lugar, com 470 espécies. O gênero Mikania, com cerca de 300 espécies, tem a maior contribuição neste somatório (Gentry 1991). Mikania tem ampla ocorrência no sul do Brasil e apresenta considerável riqueza de espécies em Floresta Ombrófila Densa no estado de Santa Catarina (Durigon et al. 2014, Citadini-Zanette et al. 2014), podendo ser explicado pelo fato de que o sul do Brasil é um dos centros de diversidade do gênero, contribuindo com espécies lenhosas nos ambientes de floresta (Ritter \& Waechter 2004). 
Quadro 1. Lista das espécies de trepadeiras encontradas no Parque Estadual da Serra Furada (PAESF), Santa Catarina, Brasil, com suas respectivas famílias, classificação quanto ao tipo de caule, mecanismo de escalada e síndrome de dispersão. * corresponde às espécies de hábito variável.

\begin{tabular}{|c|c|c|c|}
\hline Famílias/Espécies & Tipo de caule & Mecanismo de Escalada & Síndrome de Dispersão \\
\hline \multicolumn{4}{|l|}{ Acanthaceae } \\
\hline Mendoncia puberula Mart. & herbáceo & volúvel & zoocoria \\
\hline M. velloziana Mart. & herbáceo & volúvel & zoocoria \\
\hline \multicolumn{4}{|l|}{ Apocynaceae } \\
\hline Condylocarpon isthmicum (Vell.) A. DC. & lenhoso & volúvel & anemocoria \\
\hline Forsteronia thyrsoidea (Vell.) Müll.Arg. & lenhoso & volúvel & anemocoria \\
\hline F. glabrescens Müll.Arg. & lenhoso & volúvel & anemocoria \\
\hline F. rsteronia refracta Müll.Arg. & lenhoso & volúvel & anemocoria \\
\hline Gonolobus parviflorus Decne. & herbáceo & volúvel & anemocoria \\
\hline G. rostratus (Vahl) R. Br. ex Schult. & herbáceo & volúvel & anemocoria \\
\hline Orthosia scoparia (Nutt.) Liede \& Meve & herbáceo & volúvel & anemocoria \\
\hline \multicolumn{4}{|l|}{ Asteraceae } \\
\hline Baccharis anomala DC. & lenhoso & apoiante & anemocoria \\
\hline B. conyzoides DC.* & lenhoso & apoiante & anemocoria \\
\hline Cyrtocymura scorpioides (Lam.) H. Rob. & lenhoso & apoiante & anemocoria \\
\hline Mikania campanulata Gardner & herbáceo & volúvel & anemocoria \\
\hline M. cordifolia (L.f.) Willd. & herbáceo & volúvel & anemocoria \\
\hline M. glomerata Spreng. & lenhoso & volúvel & anemocoria \\
\hline M. laevigata Sch. Bip. ex Baker & lenhoso & volúvel & anemocoria \\
\hline M. lanuginosa $\mathrm{DC}$. & lenhoso & volúvel & anemocoria \\
\hline M. micrantha Kunth & herbáceo & volúvel & anemocoria \\
\hline M. cf. sericea Hook. \& Arn. & lenhoso & volúvel & anemocoria \\
\hline M. ternata (Vell.) B.L. Rob. & herbáceo & volúvel & anemocoria \\
\hline M. cf. vitifolia $\mathrm{DC}$. & herbáceo & volúvel & anemocoria \\
\hline Mutisia campanulata Less. & herbáceo & apoiante & anemocoria \\
\hline Piptocarpha sellowii (Sch. Bip.) Baker & lenhoso & apoiante & Anemocoria \\
\hline \multicolumn{4}{|l|}{ Begoniaceae } \\
\hline Begonia fruticosa A. DC.* & lenhoso & radicante & autocoria \\
\hline \multicolumn{4}{|l|}{ Bignoniaceae } \\
\hline Amphilophium crucigerum (L.) L.G. Lohmann & lenhoso & preênsil & anemocoria \\
\hline A. dolichoides (Cham.) L.G. Lohmann & lenhoso & preênsil & anemocoria \\
\hline A. dusenianum (Kraenzl.) L.G. Lohmann & lenhoso & preênsil & anemocoria \\
\hline Bignonia sciuripabulum (K. Schum.) L.G. Lohmann & lenhoso & preênsil & anemocoria \\
\hline Dolichandra quadrivalvis (Jacq.) L.G. Lohmann & lenhoso & preênsil & anemocoria \\
\hline D. unguis-cati (L.) L.G. Lohmann & lenhoso & preênsil & anemocoria \\
\hline Fridericia chica (Bonpl.) L.G. Lohmann & lenhoso & preênsil & anemocoria \\
\hline F. cf. triplinervia (Mart. ex DC.) L.G. Lohmann & lenhoso & preênsil & anemocoria \\
\hline Fridericia sp. & lenhoso & preênsil & anemocoria \\
\hline Pyrostegia venusta (Ker Gawl.) Miers & lenhoso & preênsil & anemocoria \\
\hline Tanaecium pyramidatum (Rich.) L.G. Lohmann & lenhoso & preênsil & anemocoria \\
\hline Tynanthus elegans Miers & lenhoso & preênsil & anemocoria \\
\hline \multicolumn{4}{|l|}{ Blechnaceae } \\
\hline Salpichlaena volubilis (Kaulf.) J. Sm. & herbáceo & volúvel & anemocoria \\
\hline \multicolumn{4}{|l|}{ Celastraceae } \\
\hline Pristimera celastroides (Kunth) A.C. Sm. & lenhoso & volúvel & anemocoria \\
\hline \multicolumn{4}{|l|}{ Convolvulaceae } \\
\hline Ipomoea purpurea (L.) Roth. & herbáceo & volúvel & autocoria \\
\hline I. triloba $\mathrm{L}$. & herbáceo & volúvel & autocoria \\
\hline \multicolumn{4}{|l|}{ Cucurbitaceae } \\
\hline Cayaponia palmata Cogn. & herbáceo & preênsil & zoocoria \\
\hline C. cf. ternata Cogn. & herbáceo & preênsil & zoocoria \\
\hline \multicolumn{4}{|l|}{ Dryopteridaceae } \\
\hline Mickelia scandens (Raddi) R.C. Moran et al. * & herbáceo & radicante & anemocoria \\
\hline Polybotrya cylindrica Kaulf.* & herbáceo & radicante & anemocoria \\
\hline \multicolumn{4}{|l|}{ Fabaceae } \\
\hline Dalbergia frutescens (Vell.) Britton* & lenhoso & volúvel & anemocoria \\
\hline Phanera angulosa (Vogel) Vaz & lenhoso & preênsil & anemocoria \\
\hline
\end{tabular}


Quadro 1. Cont.

\begin{tabular}{|c|c|c|c|}
\hline Famílias/Espécies & Tipo de caule & Mecanismo de Escalada & Síndrome de Dispersão \\
\hline \multicolumn{4}{|l|}{ Hymenophyllaceae } \\
\hline Vandenboschia radicans (Sw.) Copel.* & herbáceo & radicante & anemocoria \\
\hline \multicolumn{4}{|l|}{ Lomariopsidaceae } \\
\hline Lomariopsis marginata (Schrad.) Kuhn* & herbáceo & radicante & anemocoria \\
\hline \multicolumn{4}{|l|}{ Malpighiaceae } \\
\hline Banisteriopsis sp. & lenhoso & volúvel & anemocoria \\
\hline Dicella bracteosa (A. Juss.) Griseb. & lenhoso & volúvel & zoocoria \\
\hline Heteropterys aenea Griseb. & lenhoso & volúvel & anemocoria \\
\hline Peixotoa catarinensis C.E. Anderson & lenhoso & volúvel & anemocoria \\
\hline \multicolumn{4}{|l|}{ Marcgraviaceae } \\
\hline Marcgravia polyantha Delpino & lenhoso & radicante & zoocoria \\
\hline \multicolumn{4}{|l|}{ Melastomataceae } \\
\hline Pleiochiton blepharodes (DC.) Reginato et al.* & herbáceo & volúvel & zoocoria \\
\hline \multicolumn{4}{|l|}{ Onagraceae } \\
\hline Fuchsia regia (Vell.) Munz * & lenhoso & apoiante & zoocoria \\
\hline \multicolumn{4}{|l|}{ Passifloraceae } \\
\hline Passiflora capsularis $\mathrm{L}$. & herbáceo & preênsil & zoocoria \\
\hline \multicolumn{4}{|l|}{ Phytolaccaceae } \\
\hline Seguieria americana $\mathrm{L} . *$ & lenhoso & apoiante & anemocoria \\
\hline \multicolumn{4}{|l|}{ Piperaceae } \\
\hline Manekia obtusa (Miq.) T. Arias et al. & herbáceo & radicante & zoocoria \\
\hline \multicolumn{4}{|l|}{ Polygonaceae } \\
\hline Coccoloba arborescens R.A. Howard * & lenhoso & volúvel & zoocoria \\
\hline \multicolumn{4}{|l|}{ Rosaceae } \\
\hline Rubus imperialis Cham. \& Schltdl.* & lenhoso & apoiante & zoocoria \\
\hline \multicolumn{4}{|l|}{ Rubiaceae } \\
\hline Manettia luteorubra (Vell.) Benth. & herbáceo & volúvel & autocoria \\
\hline M. tweediana K. Schum. & herbáceo & volúvel & autocoria \\
\hline \multicolumn{4}{|l|}{ Sapindaceae } \\
\hline Paullinia carpopoda Cambess. & lenhoso & preênsil & zoocoria \\
\hline P. trigonia Vell. & lenhoso & preênsil & zoocoria \\
\hline Serjania multiflora Cambess. & lenhoso & preênsil & anemocoria \\
\hline Thinoua sp. & lenhoso & preênsil & anemocoria \\
\hline \multicolumn{4}{|l|}{ Smilacaceae } \\
\hline Smilax cognata Kunth & herbáceo & volúvel & zoocoria \\
\hline \multicolumn{4}{|l|}{ Violaceae } \\
\hline Anchietea pyrifolia (Mart.) G. Don & lenhoso & volúvel & anemocoria \\
\hline
\end{tabular}

Bignoniaceae possui aproximadamente 800 espécies e tem grande parte da sua diversidade no Brasil, com 56 gêneros e cerca de 338 espécies (Gentry 1980). É considerada a família com maior número de espécies de lianas no Brasil (Silva \& Queiroz 2003) e diversos estudos qualitativos apontam Bignoniaceae entre as famílias com maior número de trepadeiras (Citadini-Zanette et al. 1997, Lohmann 2006, Durigon et al. 2009, Durigon \& Waechter 2011). No presente estudo, a referida família apresentou a maior diversidade de gêneros, mas não de espécies.

Apocynaceae, a qual está entre as famílias de maior riqueza específica na área de estudo, tem grande riqueza de trepadeiras na região sul do Brasil, com cerca de 112 espécies (Durigon et al.2014). Ela também se destaca entre as famílias com maior número de espécies de trepadeiras para o estado de Santa Catarina (Citadini-Zanette et al. 2014, Durigon et al. 2014).
Quanto à ocorrência das espécies na área, Mickelia scandens (Dryopteridaceae) foi a mais abundante e com maior Índice de Valor de Importância (Tab. 1). As samambaias trepadeiras, embora representadas por apenas quatro espécies no levantamento fitossociológico, foram abundantes, correspondendo a $34 \%$ dos indivíduos registrados.

As samambaias não têm grande expressividade nos levantamentos com trepadeiras e, muitas vezes, são desconsideradas como parte do grupo, por serem confundidas com hemiepífitos, ou ainda apresentarem hábito variável. $\mathrm{Na}$ área de estudo, no entanto, constatou-se permanente conexão de todos os indivíduos com o solo, conforme também registrado por Custódio (2015). Mickelia scandens é nativa e endêmica do Brasil, ocorrendo no sul e sudeste nas áreas de Mata Atlântica (Prado \& Sylvestre 2015). 
Tabela 1. Descritores estruturais das espécies de trepadeiras amostradas no Parque Estadual da Serra Furada (PAESF), Santa Catarina, Brasil. Ni = número de indivíduos; $\mathrm{Np}$ = número de parcelas com ocorrência das espécies; $\mathrm{DA}=$ densidade absoluta; $\mathrm{DR}=$ densidade relativa; $\mathrm{FA}=$ frequência absoluta; FR = frequência relativa; IVI = índice de valor de importância.

\begin{tabular}{|c|c|c|c|c|c|c|c|}
\hline Espécies & $\mathrm{Ni}$ & $\mathrm{Np}$ & DA & DR & FA & FR & IVI \\
\hline Mickelia scandens & 67 & 22 & 111,67 & 21,47 & 36,67 & 11,64 & 16,56 \\
\hline Paullinia trigona & 37 & 18 & 61,67 & 11,86 & 30,00 & 9,52 & 10,69 \\
\hline Marcgravia polyantha & 33 & 20 & 55,00 & 10,58 & 33,33 & 10,58 & 10,58 \\
\hline Polybotria cilindrica & 20 & 5 & 33,33 & 6,41 & 8,33 & 2,65 & 4,53 \\
\hline Serjania multiflora & 18 & 13 & 30,00 & 5,77 & 21,67 & 6,88 & 6,32 \\
\hline Vandenboschia radicans & 17 & 12 & 28,33 & 5,45 & 20,00 & 6,35 & 5,90 \\
\hline Dolichandra quadrivalvis & 14 & 12 & 23,33 & 4,49 & 20,00 & 6,35 & 5,42 \\
\hline Mikania laevigata & 11 & 7 & 18,33 & 3,53 & 11,67 & 3,70 & 3,61 \\
\hline Coccoloba arborescens & 10 & 6 & 16,67 & 3,21 & 10,00 & 3,17 & 3,19 \\
\hline Begonia fruticosa & 9 & 9 & 15,00 & 2,88 & 15,00 & 4,76 & 3,82 \\
\hline Tanaecium pyramidatum & 8 & 7 & 13,33 & 2,56 & 11,67 & 3,70 & 3,13 \\
\hline Dolichandra unguis-cati & 6 & 6 & 10,00 & 1,92 & 10,00 & 3,17 & 2,55 \\
\hline Bignonia sciuripabula & 5 & 2 & 8,33 & 1,60 & 3,33 & 1,06 & 1,33 \\
\hline Phanera angulosa & 5 & 5 & 8,33 & 1,60 & 8,33 & 2,65 & 2,12 \\
\hline Dalbergia frutescens & 4 & 3 & 6,67 & 1,28 & 5,00 & 1,59 & 1,43 \\
\hline Heteropterys aenea & 4 & 3 & 6,67 & 1,28 & 5,00 & 1,59 & 1,43 \\
\hline Pristimera celastroides & 4 & 3 & 6,67 & 1,28 & 5,00 & 1,59 & 1,43 \\
\hline Amphilophium crucigerum & 3 & 3 & 5,00 & 0,96 & 5,00 & 1,59 & 1,27 \\
\hline Dicella bracteosa & 3 & 2 & 5,00 & 0,96 & 3,33 & 1,06 & 1,01 \\
\hline Forsteronia refracta & 3 & 2 & 5,00 & 0,96 & 3,33 & 1,06 & 1,01 \\
\hline Fridericia chica & 3 & 3 & 5,00 & 0,96 & 5,00 & 1,59 & 1,27 \\
\hline Manekia obtusa & 3 & 3 & 5,00 & 0,96 & 5,00 & 1,59 & 1,27 \\
\hline Smilax cognata & 3 & 2 & 5,00 & 0,96 & 3,33 & 1,06 & 1,01 \\
\hline Forsteronia glabrescens & 3 & 3 & 5,00 & 0,96 & 5,00 & 1,59 & 1,27 \\
\hline Fridericia sp. & 2 & 2 & 3,33 & 0,64 & 3,33 & 1,06 & 0,85 \\
\hline Mendoncia puberula & 2 & 1 & 3,33 & 0,64 & 1,67 & 0,53 & 0,59 \\
\hline Peixotoa catarinensis & 2 & 2 & 3,33 & 0,64 & 3,33 & 1,06 & 0,85 \\
\hline Salpichlaena volubilis & 2 & 2 & 3,33 & 0,64 & 3,33 & 1,06 & 0,85 \\
\hline Seguieria americana & 2 & 2 & 3,33 & 0,64 & 3,33 & 1,06 & 0,85 \\
\hline Amphilophium dolichoides & 1 & 1 & 1,67 & 0,32 & 1,67 & 0,53 & 0,42 \\
\hline Banisteriopsis sp. & 1 & 1 & 1,67 & 0,32 & 1,67 & 0,53 & 0,42 \\
\hline Cayaponia cf. ternata & 1 & 1 & 1,67 & 0,32 & 1,67 & 0,53 & 0,42 \\
\hline Cayaponia palmata & 1 & 1 & 1,67 & 0,32 & 1,67 & 0,53 & 0,42 \\
\hline Condylocarpon isthmicum & 1 & 1 & 1,67 & 0,32 & 1,67 & 0,53 & 0,42 \\
\hline Fridericia triplinervia & 1 & 1 & 1,67 & 0,32 & 1,67 & 0,53 & 0,42 \\
\hline Mendoncia velloziana & 1 & 1 & 1,67 & 0,32 & 1,67 & 0,53 & 0,42 \\
\hline Piptocarpha sellowii & 1 & 1 & 1,67 & 0,32 & 1,67 & 0,53 & 0,42 \\
\hline Tynanthus elegans & 1 & 1 & 1,67 & 0,32 & 1,67 & 0,53 & 0,42 \\
\hline Total & 312 & & 520 & 100 & 315 & 100 & 100 \\
\hline
\end{tabular}

Ainda entre as espécies com maior número de indivíduos e de IVI estão Paullinia trigonia (Sapindaceae) e Marcgravia polyantha (Marcgraviaceae), sendo a primeira de ampla distribuição na área e a segunda registrada nos locais mais úmidos e conservados.

Paullinia é citado como de ocorrência comum na Mata Atlântica (Somner 1993). É sugerido também, que espécies com gavinhas, como as deste gênero, estejam adaptadas a diferentes variações de disponibilidade de luz (Teramura et al. 1991), sendo muito eficientes para alcançar o dossel da floresta (Lima et al. 1997), favorecendo a sua distribuição.

Marcgravia polyantha tem sido apontada como de ambientes mais úmidos e interiores de florestas (Durigon et al. 2013), limitando-se, portanto, a ambientes mais conservados, como os encontrados neste estudo.
Embora as trepadeiras tenham sido comumente associadas a ambientes mais alterados (Gentry 1991), observou-se no presente estudo que, apesar de considerável riqueza de espécies, há baixa abundância, sendo a maioria delas representadas por menos de dez indivíduos (Tab. 1). A maior abundância corresponde às espécies radicantes, que são consideradas de ambientes bem preservados (Durigon et al. 2013). Portanto, as plantas trepadeiras integram um nicho diferenciado no ecossistema florestal, contribuindo para a riqueza da área e sugerindo que ambientes bem conservados podem ser relevantes detentores de espécies raras ou pouco abundantes (Villagra 2012, Durigon et al. 2013).

O mecanismo de escalada mais frequentemente encontrado na área de estudo foi o volúvel, com 35 espécies, 
seguido de preênsil com 21, radicante e apoiante com sete espécies cada. De maneira geral, o mecanismo volúvel destaca-se em riqueza de espécies nos levantamentos com trepadeiras (Udulutsch et al. 2004, Santos et al. 2014) e é citado como o mais representativo nas áreas subtropicais (Durigon et al. 2013). Citadini-Zanette et al. (2014) encontraram proporção de mecanismos semelhante para o estado de Santa Catarina, apenas com percentual menor de radicantes e maior de apoiantes, considerando as espécies amostradas em todas as tipologias florestais do estado, como também apenas as da Floresta Ombrófila Densa (Tab. 2).

A maior proporção de radicantes na área de estudo pode estar relacionada ao critério de inclusão, em que se amostraram tanto as lenhosas como as herbáceas dotadas deste mecanismo, além das samambaias de hábito trepador. O critério de inclusão por diâmetro, bem como a dificuldade em diferenciar as espécies hemiepífitas e trepadeiras, é apontado como limitadores para a inclusão destas espécies nos levantamentos (Villagra 2012).

Para as trepadeiras apoiantes, a dificuldade de um padrão de inclusão também ocorre por algumas espécies possuírem características de hábito variável, podendo ocorrer ou não com o hábito trepador, o que dificulta sua inclusão. No estudo de Citadini-Zanette et al. (2014) houve ainda a inclusão dos bambus apoiantes, critério diferente do adotado neste estudo.

Este estudo contribuiu para o conhecimento da riqueza e estrutura de trepadeiras no Sul do Brasil, principalmente por incluir as trepadeiras herbáceas em estudos fitossociológicos, além de ser importante por estar entre os poucos trabalhos que estudaram as trepadeiras em florestas subtropicais.

Tabela 2. Percentual de riqueza de mecanismos de ascensão das espécies trepadeiras ocorrentes na área de estudo do Parque Estadual da Serra Furada (PAESF), na amostragem para o Estado de Santa Catarina (SC) e para a Floresta Ombrófila Densa (FOD), segundo Citadini-Zanette et al. (2014).

\begin{tabular}{lccc}
\hline Mecanismos & PAESF $(\%)$ & SC (\%) & FOD (\%) \\
\hline Volúvel & 50 & 46 & 42 \\
Preênsil & 30 & 30 & 29 \\
Radicante & 10 & 4 & 4 \\
Apoiante & 10 & 20 & 25 \\
Total & 100 & 100 & 100 \\
\hline
\end{tabular}

\section{AGRADECIMENTOS}

Á Coordenação de Aperfeiçoamento de Pessoal de Nível Superior (CAPES) pela bolsa concedida à primeira autora.

\section{REFERÊNCIAS}

Angiosperm Phylogeny Group - APG IV. 2016. An update of the Angiosperm Phylogeny Group classification for the ordens and families of flowering plants. Botanical Journal of the Linnean Society181(1):1-20.
Campanili, M. \& Schäffer, W.B. 2010. Mata Atlântica: patrimônio nacional dos brasileiros. Ministério do Meio Ambiente, Brasília. 410 p.

Carneiro, J.S. \& Vieira, A.O.S. 2012. Trepadeiras: florística da Estação Ecológica do Caiuá e chave de identificação vegetativa para espécies do Norte do Estado do Paraná. Acta Scientiarum 34(2):217-223.

Citadini-Zanette, V., Soares, J.J. \& Martinello, C.M. 1997. Lianas de um remanescente florestal da microbacia do Rio Novo, Orleans, Santa Catarina Brasil. Insula 26:45-63.

Citadini-Zanette, V., Sevegnani, L., Santos, R., Gasper, A.L., Vibrans, A.C. \& Sobral, M.E.G. 2014. Plantas trepadeiras no Estado de Santa Catarina, Brasil: diversidade e distribuição. In Diversidade e conservação de trepadeiras: contribuição para a restauração de ecossistemas brasileiros (B.L.P. Villagra, M.M.R.F. Melo, S.R. Melo, \& L.M. Barbosa, eds.). Instituto de Botânica, São Paulo. 224 p.

Custódio, S.Z. 2015. Samambaias e licófitas do quadrante sul do Parque Estadual da Serra Furada, Sul de Santa Catarina, Brasil. Dissertação 158 f., Universidade do Extremo Sul Catarinense, Criciúma.

Durigon, J. 2010. Diversidade e distribuição de trepadeiras em um mosaico de ambientes florestais de um morro granítico subtropical. Dissertação 53 f., Universidade Federal do Rio Grande do Sul, Porto Alegre.

Durigon, J. 2014. Distribuição e atributos de espécies trepadeiras: análises em escala global, regional e local. Tese 216 f., Universidade Federal do Rio Grande do Sul, Porto Alegre.

Durigon, J., Durán, S.M. \& Gianoli, E. 2013. Global distribution of root climbers is positively associated with precipitation and negatively associated with seasonality. Journal of Tropical Ecology 29(4):357360.

Durigon, J., Canto-Dorow, T.S. \& Eisinger, S.M. 2009. Composição florística de trepadeiras ocorrentes em fragmentos de floresta estacional, Santa Maria, Rio Grande do Sul, Brasil. Rodriguésia 60(2):415-422.

Durigon, J., Ferreira, P.P.A., Seger, G.D.S. \& Miotto, S.T.S. 2014. Trepadeiras na Região Sul do Brasil. In Diversidade e conservação de trepadeiras: contribuição para a restauração de ecossistemas brasileiros. (B.L.P. Villagra, M.M.R.F. Melo, S.R. Melo \& L.M. Barbosa, eds.). São Paulo: Instituto de Botânica, São Paulo. 224 p.

Durigon, J., Miotto, S.T.S. \& Gianoli, E. 2014. Distribution and traits of climbing plants in subtropical and temperate South America. Journal of Vegetation Science 25(6):1484-1492.

Durigon, J. \& Waechter, J.L. 2011. Floristic composition and biogeographic relations of a subtropical assemblage of climbing plants. Biodiversity and Conservation 20(5):1027-1044.

Empresa de Pesquisa Agropecuária e de Extensão Rural de Santa Catarina - EPAGRI. 2001. Dados e informações bibliográficas da unidade de planejamento regional litoral sul catarinense-UPR8. Florianópolis. CD ROM.

Fundação do Meio Ambiente - FATMA. 2010. Plano de Manejo do Parque Estadual da Serra Furada: Plano Básico - Projeto de Proteção da Mata Atlântica em Santa Catarina. Socioambiental Consultores Associados Ltda, Florianópolis. 112 p.

Filgueiras, T.S., Nogueira, P.E., Brochado, A.L. \& Guala, G.F. 1994. Caminhamento: um método expedito para levantamentos florísticos qualitativos. Caderno de Geociências 12:39-43.

Gentry, A.H. 1980. Flora Neotropica: Bignoniaceae - Part I (Tribes Crescentieae and Tourrettieae). Flora Neotropica Monograph 25(1):1150 .

1991. The distribution and evolution of climbing plants. In The Biology of Vines (F.E. Putz \& H.A. Mooney, eds.). Cambridge University Press, Cambridge, p. 3-52.

Gerwing, J.J., Schnitzer, S.A., Burnham, R.J., Bongers, F., Chave, J., De Walt, S.J., Ewango, C.E.N., Foster, R., Kenfack, D., MartinezRamos, M., Parren, M., Parthasarathy, N., Perez-Salicrup, D.R., Putz, F.E. \& Thomas, D.W. 2006. A standard protocol for liana censuses. Biotropica 38(2):256-261.

Hegarty, E.E. 1991. Vine-host interactions. In The biology of vines (F.E. Putz \& H.A. Mooney, eds.). Cambridge University Press, Cambridge, p. 357-375.

IBGE. 2012. Fundação Instituto Brasileiro de Geografia e Estatística. Manual técnico da vegetação brasileira. IBGE, Rio de Janeiro, 275p.

IPNI. 2015. The International Plant Names Index. Disponível em: $<$ http:// www.ipni.org. Acessado em 12.01.2015. 
Lagos, A.R. \& Muller, B.L.A. 2007. Hotspot brasileiro: Mata Atlântica. Saúde e Ambiente em Revista 2(2):35-45.

Lima, H.C., Lima, M.P.M., Vaz, A.M.S.F. \& Pessoa, S.V.A. 1997. Trepadeiras da reserva ecológica de Macaé de Cima. In Serra de Macaé de Cima: Diversidade florística e conservação em Mata Atlântica (H.C. Lima \& R.R. Guedes-Bruni, eds.). Jardim Botânico do Rio de Janeiro, Rio de Janeiro, p. 75-87.

Lohmann, L.G. 2006. Untangling the phylogeny of neotropical lianas (Bignonieae, Bignoniaceae). American Journal of Botany 93:304-318.

Mueller-Dombois, D. \& Ellenberg, H. 2002. Aims and methods of vegetation ecology. The Blackburn Press, New Jersey. 547 p.

Phillips, O.L., Martinez, R.V., Arroyo, L., Baker, T.R., Killeen, T., Lewis, S.L., Malhi, Y., Mendonza, A.M., Neill, D., Vargas, P.N., Alexiades, M., Ceron, C., Di Fiore, A., Erwin, T., Jardim, A., Palacios, W., Saldias, M. \& Vincenti, B. 2002. Increasing dominance of large lianas in Amazonian forests. Nature 418:770-774.

Prado, J. \& Sylvestre, L. 2015. Pteridófitas. In Lista de Espécies da Flora do Brasil. Jardim Botânico do Rio de Janeiro. Disponível em: http:// floradobrasil.jbrj.gov.br. Acessado em 02.10.2015.

Putz, F.E. 1984. The natural history of lianas on Barro Colorado Island, Panama. Ecology 65(6):19-23.

Reis, A. 2004. Apocináceas-Asclepiadóideas. Flora Ilustrada Catarinense, Itajaí, parte $1.250 \mathrm{p}$.

Reitz, R. 1968. Apocináceas. Flora Ilustrada Catarinense. Itajaí, parte 1. $112 \mathrm{p}$.

1974. Bignoniáceas. Flora Ilustrada Catarinense. Itajaí, parte $1.172 \mathrm{p}$.

. 1980. Passifloráceas. Flora Ilustrada Catarinense. Itajaí, parte 1. $130 \mathrm{p}$.

Rezende, A.A. \& Weiser, V.L. 2014. Estudos com trepadeiras no Brasil. In Diversidade e conservação de trepadeiras: contribuição para a restauração de ecossistemas brasileiros (B.L.P. Villagra, M.M.R.F. Melo, S.R. Melo \& L.M. Barbosa, orgs.). Instituto de Botânica, São Paulo. 224 p.

Ritter, M.R. \& Waechter, J.L. 2004. Biogeografia do gênero Mikania Willd. (Asteraceae) no Rio Grande do Sul, Brasil. Acta Botanica Brasilica 18(3):643-652.

Romaniuc-Neto, S., Godoi, J.V., Villagra, B.L.P., Almeida-Scabbia, R.J. \& Melo, M.M.R.F. 2012. Caracterização florística, fitossociológica e fenológica de trepadeiras de mata ciliar da Fazenda Campininha, Mogi Guaçu, SP, Brasil. Hoenea 39(1):145-155.
Santos, E.N., Caxambu, M.G., Silva, A.R., Hoppen, M.I. \& Villagra, B.L.P. 2014. Trepadeiras da Floresta Estacional Semidecídua no Estado do Paraná, Brasil. In Diversidade e conservação de trepadeiras: contribuição para a restauração de ecossistemas brasileiros (B.L.P. Villagra, M.M.R.F. Melo, S.R. Melo \& L.M. Barbosa, orgs.). Instituto de Botânica, São Paulo. 224 p.

Silva, M.M. \& Queiroz, L.P. 2013. A família Bignoniaceae na região de Catolés, Chapada Diamantina, Bahia, Brasil. Sitientibus: Série Ciências Biológicas 3(1,2):3-21.

Sfair, J.C., Rochelle, A.L.C., Melis, J., Rezende, A.A., Weiser, V.L. \& Martins, F.R. 2015. Theoretical approaches to liana management: a search for a less harmful method. International Journal for Biodiversity Science, Ecosystem Services et Management 11(2):89-95.

Smith, A.R., Pryer, K.M., Schuettpelz, E., Korall, P., Schneider, H. \& Wolf, P.G.A. 2006. Classification for extant ferns. Taxon 55(3):705-731.

Schnitzer, S.A. \& Bongers F. 2002. The ecology of lianas and their role in forests. Trends in Ecology and Evolution 17(5): 223-230.

Somner, G.V. 1993. Duas espécies novas de Paullinia (Sapindaceae) para o sudeste do Brasil. Bradea 6(19): 167-172.

Tabanez, A.A. \& Viana, V.M. 2000. Patch structure within Brazilian Atlantic Forest fragments and implications for conservation. Biotropica 32(4):925-933.

Teramura, A.H., Gold, W.G. \& Forserth, I.N. 1991. Physiological ecology of mesic, temperate woody vines. In The Biology of Vines (F.E. Putz $\&$ H.A. Mooney, eds.). Cambridge University Press, Cambridge, p. 245-285.

Udulutsch, R.G., Assis, M.A. \& Picchi, D.G. 2004. Florística de trepadeiras numa Floresta Estacional Semidecídua, Rio Claro, Araras, Estado de São Paulo, Brasil. Revista Brasileira de Botânica 27(1):124-134.

Vibrans, A.C., Mc Roberts, R.E., Lingner, D.V., Nicoletti, A.L. \& Moser, P. 2013. Extensão original e remanescentes da Floresta Ombrófila Densa em Santa Catarina. In Inventário Florístico Florestal de Santa Catarina (A.C. Vibrans, L. Sevegnani, A.L. Gasper \& D.V. Lingner, eds.). Edifurb, Blumenau, p. 25-34.

Villagra, B.L.P. 2008. Diversidade florística e estrutura da comunidade de plantas trepadeiras no Parque Estadual das Fontes do Ipiranga, São Paulo, SP, Brasil. Dissertação 172 f., Instituto de Botânica da Secretaria de Estado do Meio Ambiente, São Paulo.

.2012. Estrutura da comunidade de trepadeiras em Mata Atlântica, Santo André, SP, Brasil. Tese 150 f., Instituto de Botânica da Secretaria de Estado do Meio Ambiente, São Paulo. 